\title{
Probabilistic assessment of transient stability in reduced inertia systems
}

DOI:

10.1109/PESGM.2016.7741651

\section{Document Version}

Accepted author manuscript

Link to publication record in Manchester Research Explorer

\section{Citation for published version (APA):}

Papadopoulos, P., Adrees, A., \& Milanovic, J. V. (2016). Probabilistic assessment of transient stability in reduced inertia systems. In Power and Energy Society General Meeting (PESGM), 2016

https://doi.org/10.1109/PESGM.2016.7741651

\section{Published in:}

Power and Energy Society General Meeting (PESGM), 2016

\section{Citing this paper}

Please note that where the full-text provided on Manchester Research Explorer is the Author Accepted Manuscript or Proof version this may differ from the final Published version. If citing, it is advised that you check and use the publisher's definitive version.

\section{General rights}

Copyright and moral rights for the publications made accessible in the Research Explorer are retained by the authors and/or other copyright owners and it is a condition of accessing publications that users recognise and abide by the legal requirements associated with these rights.

\section{Takedown policy}

If you believe that this document breaches copyright please refer to the University of Manchester's Takedown Procedures [http://man.ac.uk/04Y6Bo] or contact uml.scholarlycommunications@manchester.ac.uk providing relevant details, so we can investigate your claim.

\section{OPEN ACCESS}




\title{
Probabilistic Assessment of Transient Stability in Reduced Inertia Systems
}

\author{
Panagiotis N. Papadopoulos, Member, IEEE, Atia Adrees, Member, IEEE, Jovica V. Milanović, Fellow, IEEE \\ School of Electrical and Electronic Engineering \\ The University of Manchester \\ Manchester, UK \\ panagiotis.papadopoulos@manchester.ac.uk, atia.adrees@manchester.ac.uk, jovica.milanovic@manchester.ac.uk
}

\begin{abstract}
A probabilistic approach to assess the transient stability of power systems with increased penetration of wind and Photo-Voltaic generation is presented in this paper. The impact on transient stability due to the intermittent behavior of Distributed Energy Resources (DERs) as well as due to their dynamic response when a disturbance happens is investigated. Moreover, the effect of conventional generation disconnection and consequently inertia reduction is studied. Apart from calculating transient stability related indices, a clustering technique is also applied to provide more information considering the impact of DERs and conventional generation disconnection on transient stability.
\end{abstract}

Index Terms - distributed energy resources, clustering, inertia, probabilistic transient stability assessment, uncertainties.

\section{INTRODUCTION}

In recent years, the increasing penetration of Distributed Energy Resources (DERs) is changing the dynamic behavior of power systems. In the future, even more DER units are planned to be connected, eventually displacing conventional generators. Therefore, the need to thoroughly investigate the impact of DERs as well as the disconnection of conventional generators on system stability becomes significant [1], [2].

In general, DERs affect system stability by changing the pre-disturbance operating conditions as well as by exhibiting different dynamic response than conventional generators, during and right after disturbances. DERs utilize new technologies and are usually interfaced with the system using power electronic converters. Therefore, appropriate models of DERs need to be used to represent their dynamic behavior. Recently, [3], [4] and [5] provided appropriate models for use in large scale stability studies, including the representation of all relevant controllers. The intermittent nature of most of the DER units such as wind generators and Photo-Voltaic (PV) units is introducing higher uncertainty in determining pre-fault operating conditions raising also the need for evaluation of worst case operating conditions [6]. Probabilistic transient stability assessment has proven to be an appropriate way to study these systems [7], as it can adequately account for uncertain parameters affecting their behavior, e.g., system loading, fault location, fault duration, etc. [8]. In [9], the uncertainty of wind and other DERs is considered for the purpose of online Dynamic Security Assessment while [10] focuses on the effect of wind uncertainty on transient stability.

In this paper, the impact of both wind and PV units on system transient stability is investigated. Apart from the aforementioned uncertainties, the disconnection of conventional generation due to DER penetration is also considered. Monte Carlo (MC) dynamic simulations for several test cases are carried out and statistical measures of transient stability indices are presented. Furthermore, for different level of penetration of DERs the conventional generators are clustered based on their post disturbance dynamic behavior [8] as change in synchronous generator grouping patterns provides additional information about the effect of DERs on overall system transient behavior.

\section{Methodology}

Fig. 1 shows the steps followed to assess the transient stability of power systems in a probabilistic manner. An appropriate dynamic model of the system, including DER units, is obtained first. Afterwards, the uncertainties related to system loading, wind and PV generation, fault location and fault duration are considered, using appropriate Probability Distribution Functions (PDFs). Then an Optimal Power Flow (OPF) problem is solved to determine the power dispatch of conventional generators. The resulting generation dispatch helps determine the amount of required disconnection of conventional generation. Finally a number $\left(N_{s}\right)$ of $\mathrm{MC}$ dynamic simulations are executed and the rotor angles of synchronous generators are obtained. In this study $N_{s}$ is set to 1000 which by trials is considered enough to reveal the underlying tendencies. (The trials were made for up to 6000 simulations for some cases and the results were not leading to different conclusions.) Increase in the number of $\mathrm{MC}$ simulations provides more detailed results. However, there is substantial increase in computational time without revealing substantially different behavior. Using the resulting rotor 


\section{$>$ ACCEPTED VERSION OF THE PAPER <}

angle values, transient stability indices are calculated to assess the transient stability status of the system. Furthermore, clustering is used to determine generator grouping patterns [8].

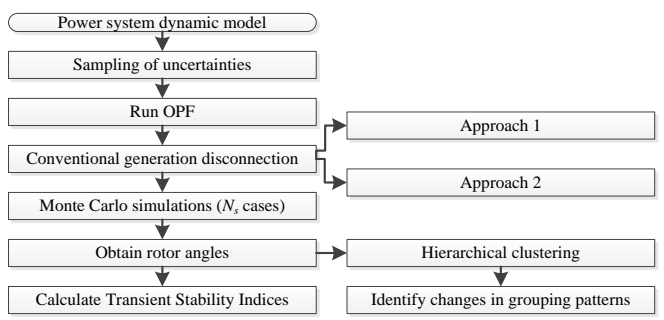

Figure 1. Flowchart of the proposed probabilistic approach.

\section{A. System under Study}

The test network used, is a modified version of the IEEE 68 bus/16 machine reduced order equivalent model of the New England Test System and the New York Power System (NETS - NYPS). The system consists of five areas, NETS, NYPS and three external areas represented by G14, G15 and G16 respectively. The conventional part of the test network is adopted from [12] and ten DER units are connected at the buses shown in Fig. 2. For each DER unit, two types of DERs are connected at each bus: Type 3 Doubly Fed Induction Generators (DFIGs), representing wind generators and Type 4 Full Converter Connected (FCC) units, representing both wind generators and Photo-Voltaic (PV) units. The models used are available in DIgSILENT/PowerFactory [13] software and are suitable generic RMS models for large scale stability studies following a similar approach to [3]-[5]. Studies with IEC 61400 models [5] have also been conducted providing similar results and leading to the same conclusions. More information on the modified test network and DER modelling can be found in [14]. All DER units are considered to provide Fault Ride Through (FRT) capability. The whole system is modeled using RMS simulations in DIgSILENT/PowerFactory.

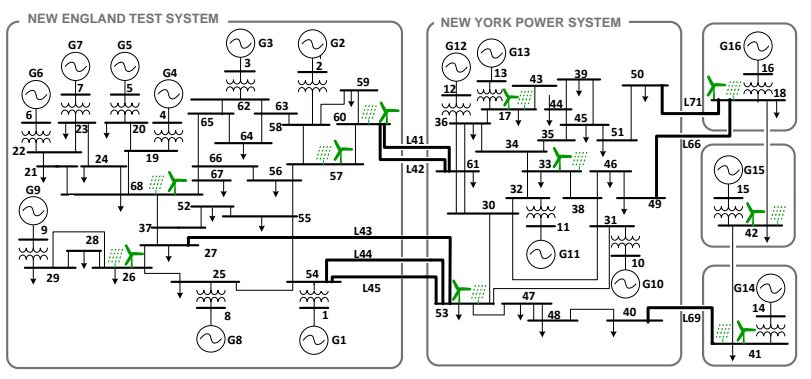

Figure 2. Modified IEEE 68 bus system.

\section{B. Uncertainties Modeling}

The two most common types of DERs are wind generators and Photo-Voltaic (PV) units. The effect of the intermittent behavior of these two primary energy sources is therefore modeled using appropriate probability distributions [10]-[11]. The uncertainties related to the conventional part of the system such as system loading, fault location and duration are also included in this study.

For system loading and PV generation, typical daily curves are initially used, obtained from national grid data [15] and the literature [11], respectively. The system load is considered to vary from 0.6 to $1 \mathrm{pu}$. The hour of the day is sampled randomly and for each hour, an appropriate PDF is used to model the uncertainty within the hour. For system loading a normal distribution with mean value $1 \mathrm{pu}$ and standard deviation $3.33 \%$ is used [8], while for PV generation, a beta distribution with $a$ and $b$ parameters 13.7 and 1.3 [16]. For wind generation, a Weibull distribution is used with $\varphi=11.1$ and $k=2.2$ [17], assuming constant wind speed for every hour within the day. For each load within the system and each DER unit, the PDFs are sampled separately, to consider independent behavior of each unit. For example, assuming the total load is $0.8 \mathrm{pu}$ for a given hour of the day, one load might have a value of $0.75 \mathrm{pu}$ and another $0.85 \mathrm{pu}$. The fault location is also sampled randomly following a uniform distribution along each line within the system. Moreover, the uncertainty of the fault duration is modeled using a normal distribution with mean value 13 cycles and standard deviation $6.67 \%$ [8]. Three phase self-clearing faults are considered as disturbances in all simulated cases.

\section{Conventional Generation Disconnection}

In order to model the disconnection of conventional generation, two main approaches are used in this paper. To describe the differences between the two approaches the spare capacity $S C_{i g}$, the instantaneous penetration level $P L_{i a}$ and the amount of conventional generation disconnection $S R_{i a}$ are defined in (1)-(3). The subscript $i=1 \ldots 1000$ denotes the dynamic simulation number, $a=1 \ldots .5$ the system area, $g$ the generator number and $d$ the DER unit number.

$$
\begin{gathered}
S C_{i g}=1-\frac{P_{S G, i g}}{S_{S G, i g} \cdot p f_{S G, i g}} \\
P L_{i a}=\frac{\sum_{n=1}^{d} P_{D E R, i n}}{\sum_{m=1}^{g} P_{S G, i m}+\sum_{n=1}^{d} P_{D E R, i n}} \\
S R_{i a}=1-\sum_{m=1}^{g} \frac{S_{S G, i m}}{S_{S G, i m}^{0}}
\end{gathered}
$$

where $P_{S G, i g}$ is the power produced by each generator (determined by OPF), $S_{S G, i g}$ is the apparent power of each generator after considering any disconnection considering $p f_{S G, i g}$ power factor, $P_{D E R, i d}$ and $S_{D E R, i d}$ are the active and apparent power produced by each DER unit (both DFIGs and FCC units) and $S_{S G, i g}{ }^{0}$ is the initial nominal apparent power of each generator before any disconnection is considered.

In the first approach, all generators (G1-G16) are considered as equivalent generators and their nominal apparent power $S_{S G, i g}$ is reduced to represent conventional generation disconnection. Since the model parameters are in pu values, this is equivalent to reducing the moment of inertia as well as increasing the actual reactance values of the generators in Ohms. In the second approach, some of the generators (G1-G16) within the test network are disconnected. Therefore, in the first approach $S_{S G, i g}$ is changing from the initial value of $S_{S G, i g}{ }^{0}$ leading to conventional generation disconnection of $S R_{i a}$ in each of the different areas of the system. On the other hand, following the second approach $S_{S G, i g}$ is equal to $S_{S G, i g}{ }^{0}$ for the conventional generators 


\section{$>$ ACCEPTED VERSION OF THE PAPER <}

remaining connected. However, $S R_{i a}$ still changes due to full disconnection of some of the generators. In both approaches, the instantaneous penetration level $P L_{i a}$ is changing due to the intermittency of DERs and to the active power output changes of each generator after solving OPF. Considering the first approach, the amount of reduction in apparent power can be either a constant percentage, or it can be considered to vary within the day according with the changes in the system load and DER unit production. In case the reduction is constant, $S C_{i g}$ varies and $S R_{i a}$ is kept constant. This is similar to the second approach and provides a direct comparison between them. Alternatively $S C_{i g}$ can be kept constant for all simulated cases leading to variation in $S R_{i a}$. Keeping $S C_{i g}$ constant for all simulated cases assumes that the system operator is always targeting at keeping a specific percentage of additional conventional generation available.

\section{Transient Stability Index}

In order to identify the impact of DERs on transient stability, the Transient Stability Index (TSI) is used, shown in (4), where $\delta_{\max , i}$ is the maximum separation between the rotor angles of any two generators for the same time instance [18]. This index provides a general overview of the overall system for the specific contingency. Negative values of the index mean that the separation between the rotor angles of two generators is greater than 360 degrees and the system is considered unstable. On the other hand, a more positive value means that the system is more stable. Apart from the TSI, the maximum rotor angle deviation for each generator, is calculated as a generator specific measure.

$$
T S I_{i}=100 \cdot \frac{360-\delta_{\max , i}}{360+\delta_{\max , i}}
$$

\section{E. Clustering}

In order to provide a more complete analysis of the impact of DER units and disconnection of conventional generation on system stability, the generator grouping patterns for the unstable cases are also determined according to the methodology described in [8]. Hierarchical clustering is applied on the generator rotor angle values, at 1.5 seconds after the fault is cleared. The Euclidean distance is used to measure the similarity between clusters and the cutoff value to form the clusters is chosen as 360 degree, as in [8]. This way the generators are categorized in groups where at least one generator of each group has 360 degrees difference from at least one generator of the other groups. By observing the changes in generator grouping patterns, important information can be obtained, such as specific increase/decrease in number of instabilities related to certain generators.

\section{PROBABILISTIC TRANSIENT STABILITY ASSESSMENT}

Ten Test Cases (TCs) are presented in total in this paper. The first five (TC1-TC5) are used to show the impact of different ways of representing conventional generation disconnection, while TC6-TC10 are used to show the impact of high penetration of DERs and consequently increased amount of disconnected conventional generation. In all TCs that include DERs, the percentage in the TC description refers to the nominal/maximum available power from DERs $\left(P_{D E R, i d}{ }^{0}\right)$ and is calculated based on the initial total generation
$S_{S G, i g}{ }^{0}$ for each area of the system. This is different to $P L_{i a}$ described above, which is the actual penetration of the specific instant based on operating power of both conventional generation and DERs for each one of the $1000 \mathrm{MC}$ simulations carried out.

In TC1, there are no DERs connected in the network and no conventional generation disconnection is considered. In this case, the generators are only de-loaded, leading to varying spare capacity $S C_{i g}$. In TC2, 20\% nominal/maximum capacity of DERs based on $S_{S G, i g}{ }^{\circ}$ for each area is added with no conventional generation disconnection. TC3 is based on TC2 but the first approach for inertia reduction is also applied by reducing $S_{S G, i g}$ of all generators by a fixed amount of $10 \%$. In TC4 the second approach for inertia reduction is applied and the entire G2, G10 and G15 are disconnected, leading to slightly more than $10 \%$ inertia reduction. In TC5, the first approach for inertia reduction is used but for each one of the $1000 \mathrm{MC}$ simulations the spare capacity of conventional generation is considered constant at $15 \%$. To sum up, TC1 and TC2 have $S R_{i a}$ equal to 0 and $S C_{i g}$ varies, TC3 and TC4 have $S R_{i a}$ constant at around $10 \%$ and $S C_{i g}$ varies and TC5 has $S C_{i g}$ constant and $S R_{i a}$ varies. In TC6, the same approach for inertia reduction with TC5 is followed but no DERs are present in the system. Therefore all the generation disconnection is caused only by load variations. TC7 to TC10 follow the same approach as TC5 but the nominal/maximum connected DER generation is increased to $30 \%, 40 \%, 50 \%$ and $60 \%$, respectively, based on $S_{S G, i g}{ }^{0}$.

\section{A. Overview of TCs using hierarchical clustering results}

In Table I, the total number of unstable cases and unstable generator grouping patterns, out of 1000 simulations for each TC, is presented. Moreover, the three most common patterns are also provided along with their percentage of occurrence (out of the total unstable cases). The highest number of unstable cases is observed in TC6 where there are no DERs and conventional generators are disconnected by keeping the spare capacity to $15 \%$. This suggests that adding DERs while still keeping spare capacity constant at $15 \%$ (TC5) can under circumstances improve the transient stability of the system. The most stable case is TC2 with DERs connected and no conventional generation disconnection considered, i.e. all SG are connected but operating at reduced output to accommodate DERs. The loading level variation is kept the same between TCs to highlight only the impact of DER penetration and disconnection of conventional generation. The most common unstable patterns are related with G9 and G11 losing stability separately. When the first approach considering conventional generation disconnection is followed, a large number of patterns with only a few cases each are observed. Moreover, the pattern related to G9 loosing stability becomes more frequent, compared to the rest of the cases (TC1-TC4).

In the literature both positive and negative impact of DERs has been documented due to various mechanisms [10], [19][20]. Reactive power support from DER units providing FRT capability usually improves transient stability [20]. However, the topology of each network, the DER location and consequent disconnection of conventional generators as well as their control strategy can affect transient stability in either a positive or negative manner [19]. Out of the 1000 simulated 


\section{$>$ ACCEPTED VERSION OF THE PAPER <}

contingencies for each test case transient stability improves for some while it deteriorates for others. For example, some cases with DERs are more stable than without DERs and vice versa. For this reason, it is important to follow a probabilistic approach in order to obtain a better overall transient stability assessment. For this specific network topology, connection of DERs tends to improve transient stability, while disconnecting conventional generation following any of the two approaches, tends to increase the number of unstable cases. These are two opposing effects which together determine the overall system behavior. While for the specific system the positive effect is dominant until around 50\% penetration (TC9), this might not necessarily be true for different network topologies and/or DER locations. Moreover, an opposite effect is observed for certain generators in the case of high amount of connected DERs (60\%-TC10). Mainly G9 is affected, exhibiting an increase in the number of instabilities compared to 50\%-TC9, as shown in Fig. 3. This is attributed to the amount of conventional generation disconnection which after a point might overcome the positive impact of DER FRT control.

TABLE I. HIERARCHICAL ClUSTERING RESUlTS

\begin{tabular}{|c|c|c|c|c|c|}
\hline & TC1 & TC2 & TC3 & TC4 & TC5 \\
\hline Unst. cases & 37 & 18 & 32 & 57 & 98 \\
\hline Patterns & 3 & 3 & 3 & 4 & 14 \\
\hline 1st unst. & $(\mathrm{G} 9)$ & $(\mathrm{G} 11)$ & $(\mathrm{G} 11)$ & $(\mathrm{G} 11)$ & $(\mathrm{G} 9)$ \\
group & $45.95 \%$ & $55.56 \%$ & $46.88 \%$ & $52.63 \%$ & $54.08 \%$ \\
\hline $\begin{array}{c}\text { 2nd unst. } \\
\text { group }\end{array}$ & $45.95 \%$ & $38.89 \%$ & $40.63 \%$ & $42.11 \%$ & $19.39 \%$ \\
\hline 3rd unst. & $(\mathrm{G} 10)$ & $(\mathrm{G} 10)$ & $(\mathrm{G} 10)$ & $(\mathrm{G} 4-\mathrm{G} 7)$ & $(\mathrm{G} 4-\mathrm{G} 5)$ \\
group & $8.11 \%$ & $5.56 \%$ & $12.5 \%$ & $1.75 \%$ & $4.08 \%$ \\
\hline & TC6 & TC7 & TC8 & TC9 & TC10 \\
\hline Unst. cases & 116 & 73 & 76 & 66 & 90 \\
\hline Patterns & 16 & 13 & 13 & 12 & 9 \\
\hline 1st unst. & $(\mathrm{G} 9)$ & $(\mathrm{G} 9)$ & $(\mathrm{G} 9)$ & $(\mathrm{G} 9)$ & $(\mathrm{G} 9)$ \\
group & $47.41 \%$ & $43.84 \%$ & $50 \%$ & $56.06 \%$ & $56.67 \%$ \\
\hline 2nd unst. & $(\mathrm{G} 11)$ & $(\mathrm{G} 11)$ & $(\mathrm{G} 11)$ & $(\mathrm{G} 11)$ & $(\mathrm{G} 11)$ \\
group & $17.24 \%$ & $27.40 \%$ & $25 \%$ & $25.76 \%$ & $23.33 \%$ \\
\hline 3rd unst. & $(\mathrm{G} 2-\mathrm{G} 9)$ & $(\mathrm{G} 4-\mathrm{G} 5)$ & $(\mathrm{G} 4-\mathrm{G} 5)$ & $(\mathrm{G} 4-\mathrm{G} 5)$ & $(\mathrm{G} 4-\mathrm{G} 5)$ \\
group & $7.76 \%$ & $9.59 \%$ & $5.26 \%$ & $3.03 \%$ & $6.67 \%$ \\
\hline
\end{tabular}

\section{B. Investigation of Conventional Generation Disconnection}

In Fig. 3 the number of cases that each specific generator exhibits instability is shown for some representative cases. While in general, TC5 is the most unstable case (from TC1TC5), G11 presents higher number of instabilities in TC4, revealing that local network characteristics can cause adverse effects compared to the general trend. The same applies for G11 between TC5 and TC6. It is therefore significant to investigate individual generator behavior as well.

The Cumulative Distribution Function (CDF) of the spare capacity of the whole NETS area is presented in Fig. 4a for TC1-TC4. TC2, which presents the smallest number of instabilities, has the largest spare capacity. This is expected since part of the load is covered by DERs and therefore, conventional generators are more de-loaded leading to increased stability. More specifically, there is a $50 \%$ probability that spare capacity is smaller than around $30 \%$ for TC1, TC3 and TC4 while for TC2 it is around 38\%. TC4 presents slightly lower spare capacity than TC3 which combined with local topological effects due to full disconnection of $\mathrm{G} 2$ and G10, lead to increased number of instabilities. In order to highlight the effect of spare capacity, the maximum rotor angle deviation of G9 is plotted against the spare capacity of the generator for TC1-TC3, in Fig. 4b. Most of the unstable cases, exhibiting high angle deviation, are observed for low values of spare capacity, i.e. when the generator is more heavily loaded. The CDF of the TSI for TC1-TC5 is also presented in Fig. 5a. The probability of the TSI to have negative values highlights the number of unstable cases. For positive TSI values, TC5 which is the approach with most unstable cases, exhibits also in general lower TSI values. For example, for TC5 there is a $50 \%$ probability that TSI will exhibit values lower than 50, while for the rest of the cases it is around 55-60. The same applies with TC4, when compared to TC3, indicating that the system tends to be less stable when full disconnection of certain generators is considered.

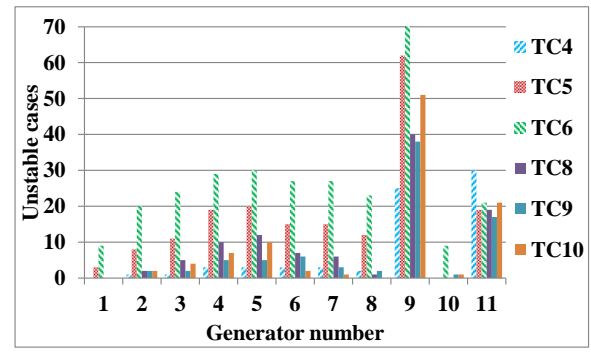

Figure 3. Unstable cases for each generator for TC4-TC6 and TC8-TC10.
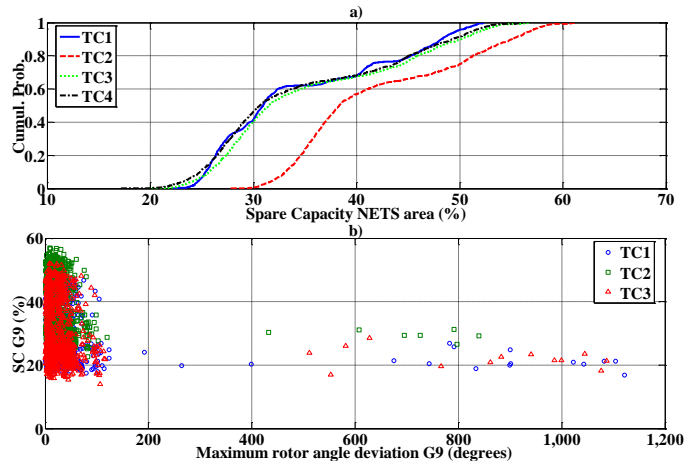

Figure 4. a) Spare capacity in NETS, b) Variation of maximum rotor angle deviation of G9 with spare capacity

\section{Investigation of Increased Penetration}

In Fig. 6a, the amount of conventional generation disconnection is plotted against the DER penetration level for NETS area for TC5 and TC10. There is a linear trend between the two variables, i.e. as the penetration level increases the amount of conventional generation disconnection is also increasing. However, the values are dispersed for specific penetration levels within a range of approximately $15 \%$ due to different system loading level, which varies from approximately 0.6 to $1 \mathrm{pu}$. In Fig. 6b, the CDFs of conventional generation disconnection for different penetration levels are presented. As the penetration level increases, the amount of conventional generation disconnection generally increases. The amount of disconnection varies from $15 \%$ to $54 \%$ for TC5, from $21 \%$ to $71 \%$ for TC9 and from $23 \%$ to $77 \%$ for TC10. Moreover, from the CDFs it is shown that there is a $40 \%$ probability that the amount of conventional generation disconnection is larger 


\section{$>$ ACCEPTED VERSION OF THE PAPER <}

than $20 \%$ for TC6 and it becomes $50 \%$ for TC10. The shape of the CDFs is also changing as DER penetration increases. In TC6 the only variable affecting generation disconnection is the system loading. From TC5 to TC10, the curve is slowly changing shape, indicating the increasing impact of DER intermittency on conventional generation disconnection.

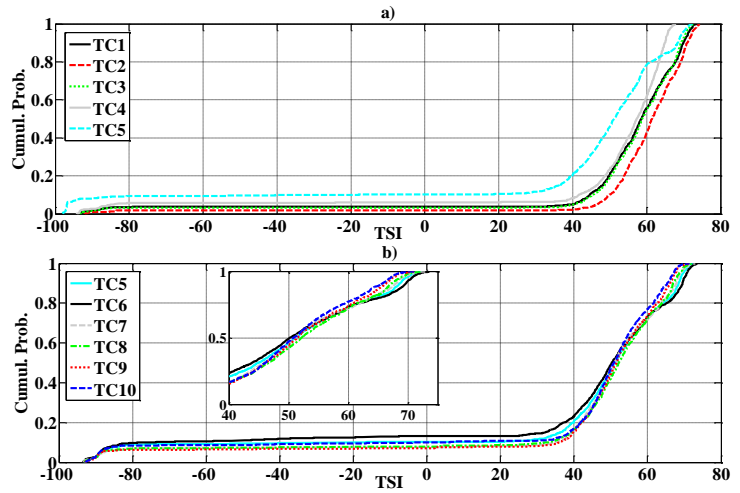

Figure 5. CDF of TSI for a) TC1-TC5 and b) TC5-TC10

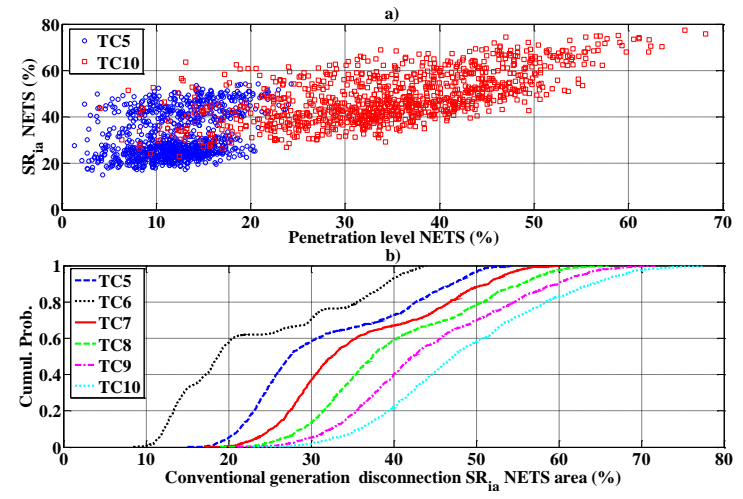

Figure 6. a) Variation of $S R_{i a}$ with penetration and b) CDF of $S R_{i a}$

A more detailed overview can be provided by observing the CDF of the TSI for TC5-TC10 in Fig. 5b. TC6 has the largest probability for the TSI to exhibit a negative value followed by TC5, TC10, TC7 and TC8. Until TC9, transient stability improves in general as the amount of connected DERs increases. However, for TC10 the number of unstable cases increases but it still remains lower than TC5 and TC6. Moreover, as penetration of DERs increases, TSI values above 60 , tend to decrease suggesting the occurrence of higher amplitude oscillations.

\section{CONCLUSIONS}

The purpose of the proposed probabilistic methodology was to identify the overall impact of DERs on power system transient stability and to provide an insight into possible constraints during decommissioning of conventional generators either at the planning or operational phase. The impact of different ways of disconnection of conventional generation is investigated taking also into consideration the impact of the uncertainties introduced by wind and PV units. While DERs offering FRT capability can improve transient stability, the disconnection of conventional generation has adverse effect. It depends on the specific system configuration and location and control of DERs and conventional generators to determine whether the overall impact is going to be positive or negative and at which DERs penetration level this impact might change. For the specific network studied, this occurs at around $50 \%$ penetration level. Apart from system wide measures and indices, it is important for generator specific indices to be introduced to identify local tendencies. Transient stability of specific generators might deteriorate while the stability of others might improve. Identifying generator grouping patterns using hierarchical clustering provides additional information in this respect.

\section{REFERENCES}

[1] V. Trovato, S. H. Tindemans, and G. Strbac, "Demand response contribution to effective inertia for system security in the GB 2020 gone green scenario," 4th ISGT Eur., Denmark, October, 2013.

[2] L. Roberts, G. Kafanas, A. Champneys, M. di Bernardo, K. Bell, "A parametric investigation on the effects of inertia on the stability of power systems," PowerTech 2015, Eindhoven, June 29 -July 2, 2015.

[3] WECC Wind Power Plant Dynamic Modeling Guide, WECC Renewable Energy Modeling Task Force, January 2014.

[4] WECC PV Power Plant Dynamic Modeling Guide, WECC Renewable Energy Modeling Task Force, May 2014.

[5] Wind turbines - Part 27-1: Electrical simulation models - Wind turbines, IEC 61400-27-1, 2015.

[6] R. Preece and J. V Milanović, "Assessing the Applicability of Uncertainty Importance Measures for Power System Studies," IEEE Trans. Power Syst., 2015, available online.

[7] W. Li, "Probabilistic Transient Stability Assessment," in Risk Assessment of Power Systems: Models, Methods, and Applications, Wiley-IEEE Press, 2014.

[8] T. Guo, J. V Milanović, "Online Identification of Power System Dynamic Signature Using PMU Measurements and Data Mining," IEEE Trans. Power Syst, 2015, available online.

[9] P. Lund, C. L. Bak, P. Thogersen, Z. Chen, C. Liu, K. Sun, and Z. H. Rather, "A Systematic Approach for Dynamic Security Assessment and the Corresponding Preventive Control Scheme Based on Decision Trees," IEEE Trans. Power Syst., vol. 29, no. 2, pp. 717-730, 2014.

[10] L. Shi, S. Sun, L. Yao, Y. Ni, and M. Bazargan, "Effects of wind generation intermittency and volatility on power system transient stability," IET Renew. Power Gener., vol. 8, no. 5, pp. 509-521, 2014.

[11] M. Fan, V. Vittal, G. T. Heydt, R. Ayyanar, "Probabilistic power flow analysis with generation dispatch including photovoltaic resources," IEEE Trans. Power Syst., vol. 28, no. 2, pp. 1797-1805, 2013.

[12] G. Rogers, Power System Oscillations. Kluwer Academic, 2000.

[13] DIgSILENT-PowerFactory User Manual, DIgSILENT GmbH, 2014.

[14] P. N. Papadopoulos, T. Guo, X. Wang, J. V Milanovic, "Impact of Penetration of Non-Synchronous Generators on Power System Dynamics," PowerTech 2015, Eindhoven, June-July, 2015.

[15] Daily load curve data, National Grid, http://www2.nationalgrid.com/UK/Industry-information/Electricitytransmission-operational-data/Data-Explorer/.

[16] Shi Tao, Yu Ruoying, Zhu Lingzhi, Gao Shan, "Power system probabilistic production simulation containing large-scale wind power and photovoltaic power," in IEEE Asia-Pacific Power and Energy Engineering Conference (APPEEC), Beijing, 8-11 Dec. 2013.

[17] R. Preece and J. V. Milanovic, "Tuning of a damping controller for multiterminal VSC-HVDC grids using the probabilistic collocation Method," IEEE Trans. Power Deliv., vol. 29, no. 1, pp. 318-326, 2014.

[18] "DSA Tools TSAT User Manual," Powertech Labs Inc, 2011.

[19] S. Eftekharnejad, V. Vittal, G. T. Heydt, B. Keel, and J. Loehr, "Impact of Increased Penetration of Photovoltaic Generation on Power Systems," Power Syst. IEEE Trans., vol. 28, no. 2, pp. 893-901, 2013.

[20] E. Vittal, M. O'Malley, A. Keane, "Rotor angle stability with high penetrations of wind generation," IEEE Trans. Power Syst., vol. 27, no. 1, pp. 353-362, 2012. 